\title{
Vorschläge zum künftigen Vorgehen bei der Zertifizierung
}

\author{
Martin Wilhelm
}

Med. Klinik 5, Klinikum Nürnberg, Nürnberg, Deutschland

Die Zertifizierung als onkologisches Zentrum ist ein aufwendiges Vorhaben, das von allen daran Beteiligten viel Engagement erfordert - und darum auch als gemeinsame Aufgabe aller Mitarbeiter verstanden werden sollte. Das setzt voraus, dass die Mitarbeiter vom Sinn und Nutzen der angestrebten Zertifizierung überzeugt sind. Ein wichtiges Argument für die Zertifizierung lässt sich allein schon aus der demographischen Entwicklung der Bevölkerung ableiten: Der wachsende Anteil älterer Menschen wird zu einem deutlichen Anstieg an Krebsneuerkrankungen führen. Der damit verbundene erhöhte Bedarf an Therapieplätzen wird sich eher einfordern lassen, wenn die Klinik einen Kompetenznachweis in Form einer Zertifizierung vorweisen kann. Auch die stetig wachsenden Kosten für die onkologische Versorgung erfordern eine Optimierung der Abläufe. Ein weiterer Vorteil der Zentrumsbildung liegt in der besseren Strukturierung der Abläufe und der Nutzung von Synergien, etwa durch Bildung von interdisziplinären Tumorboards. Langfristig ergeben sich durch die Zertifizierung nach unserer Erfahrung eindeutige Qualitätsvorteile und damit letztlich eine zusätzliche Sicherheit für die Patienten. Die schnelle Diagnosestellung sowie das abgestimmte Vorgehen aller beteiligten Fachdisziplinären ist nicht nur ein Qualitätsmerkmal, sondern wird von den Patienten auch als Servicevorteil wahrgenommen. Im Hinblick auf das Marketing geht von einer Zertifizierung eine nicht zu unterschätzende Signalwirkung aus. Letztlich muss das Vorhaben von den Schaltstellen in der Verwaltung, der Ärzteschaft und dem Pflegedienst vorbehaltlos getragen werden, wofür unter Umständen viel Überzeugungsarbeit zu leisten ist.

Auf dem Weg zur Zertifizierung ist zunächst zu klären, welche Art von Zentrum - Organkrebszentrum, Onkologisches Zentrum oder Onkologisches Spitzenzentrum - eingerichtet werden soll. Fällt die Entscheidung für ein (die Einzelorganerkrankungen überschreitendes) Onkologisches Zent- rum, bieten sich hierfür verschiedene Zertifizierungsmodelle an. Dass diese sowohl von der Deutschen Gesellschaft für Hämatologie und Onkologie (DGHO) als auch von der Deutschen Krebsgesellschaft (DKG) angeboten werden, ist aus unserer Sicht eher nachteilig. Hindernisse auf dem Weg zu einem einheitlichen Zertifizierungsmodus sind auch ein zu starkes fach- und sektorbezogenes Denken sowie divergierende Interessen der verschiedenen Fachgesellschaften. Auch der hohe Kosten- und Personalaufwand stehen der Bildung weiterer Onkologischer Zentren im Wege.

\section{Ein normatives Dokument der Qualitätsanforderungen}

Wir wünschen uns zuvorderst einen allgemein gültigen, öffentlich zugänglichen Katalog, der die Qualitätsanforderungen für eine Zertifizierung definiert. Ein solch normatives Dokument sollte den Charakter eines «Grundsatzpapiers» haben, sodass keiner der Anbieter Exklusivrechte geltend machen kann (Abb. 1).

Schwierigkeiten bei der Etablierung Onkologischer Zentren ergeben sich unter anderem aus der häufig unklaren Position des Hämatologen/Onkologen sowohl innerhalb des Gesamtkonstruktes als auch in Abgrenzung zu den Organkrebszentren. Auch der Zertifizierungsmodus an sich kann - zumindest phasenweise - zu Unmut führen, etwa wenn Fachpersonal für Monate mit der Vorbereitung des Zertifizierungsverfahrens beschäftigt ist und damit für anderweitige Aufgaben nicht zur Verfügung steht. Hier muss die Frage erlaubt sein, ob stets alle Anforderungen nach DIN EN ISO 9001 zu erfüllen sind oder ob nicht für den Bereich Onkologie (in Abgrenzung zu anderen Managementsystemen) eine analoge Zertifizierung mit den wesentlichen Qualitätsmerkmalen (wie z.B. Qualitätshandbuch, Standard Operating Procedure (SOP) der wichtigsten Behandlungsab-

\begin{tabular}{ll}
\hline KARGER & ( 2012 S. Karger GmbH, Freiburg \\
0378-584X/12/3516-0018\$38.00/0 \\
$\begin{array}{l}\text { Fax+49 7614520714 } \\
\text { Information@Karger.de } \\
\text { www.karger.com }\end{array}$ & $\begin{array}{l}\text { Accessible online at: } \\
\text { www.karger.com/onk }\end{array}$
\end{tabular}


läufe usw.) genügen. Man muss dabei bedenken, dass die Kosten für diesen zusätzlichen Aufwand zum großen Teil aus den Erlösen für die Patientenversorgung bestritten werden müssen.

Die obligatorische Bildung von Organzentren innerhalb eines Onkologischen Zentrums (DKG-Modell) sollte ebenfalls kritisch hinterfragt werden. Jedes dieser Organzentren verfügt über eigene Strukturen, was zu einer Redundanz bei den Abläufen führt und damit hohe Kosten verursacht. Eine aus unserer Sicht praktikable Alternative ist die Gründung eines Onkologischen Zentrums nach dem DGHO-Modell mit Einbindung von Organzentren in Form von engen strukturellen Kooperationen.

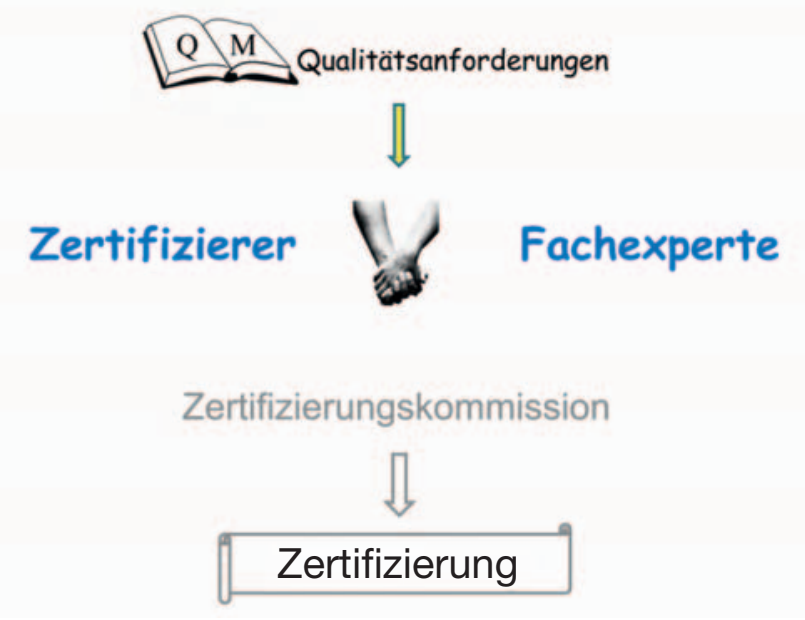

Abb. 1. Vorschlag zum Vorgehen.

\section{Zertifizierer und Fachexperte: Im Idealfall Hand in Hand}

Auf der Basis des beschriebenen Grundsatzpapiers, das die Qualitätsanforderungen im Rahmen einer Zertifizierung unabhängig vom Zertifikataussteller definiert, sollten Zertifizierer und Fachexperten gemeinsam tätig werden. In diesem Zusammenhang sind sowohl vom Zertifizierer als auch vom Fachexperten bestimmte Forderungen zu erfüllen:

Der Zertifizierer sollte

- durch den Antragsteller frei wählbar sein,

- nicht exklusiv für eine Fachgesellschaft arbeiten,

- einer unabhängigen externen Institution angehören,

- vorab keine Beratungsleistungen in Rechnung stellen,

- durch die Deutsche Akkreditierungsstelle (DAkkS) akkrediert sein und

- als Verantwortlicher über die Erteilung/Verweigerung des Zertifikates entscheiden.

Der Fachexperte sollte

- nach definierten Kriterien («Geschäftsordnung») ernannt werden,

- beratend tätig sein, jedoch auf Augenhöhe mit dem Zertifizierer,

- regelmäßig an Schulungen teilnehmen,

- durch Fachgesellschaften anerkannt sein,

- in einem allgemein zugänglichen «Fachexperten-Verzeichnis» gelistet sein.

Darüber hinaus befürworten wir die Einrichtung einer Zertifizierungskommission, wie diese im Zertifizierungsschema der DGHO bereits integriert ist. Die Zertifizierungskommission hat eine übergeordnete Funktion und kann in strittigen Fällen eingeschaltet werden. Unseres Erachtens sollte es sich bei die-

\begin{abstract}
Abb. 2. Organisationsstruktur des IOZ Klinikum Nürnberg.
\end{abstract}

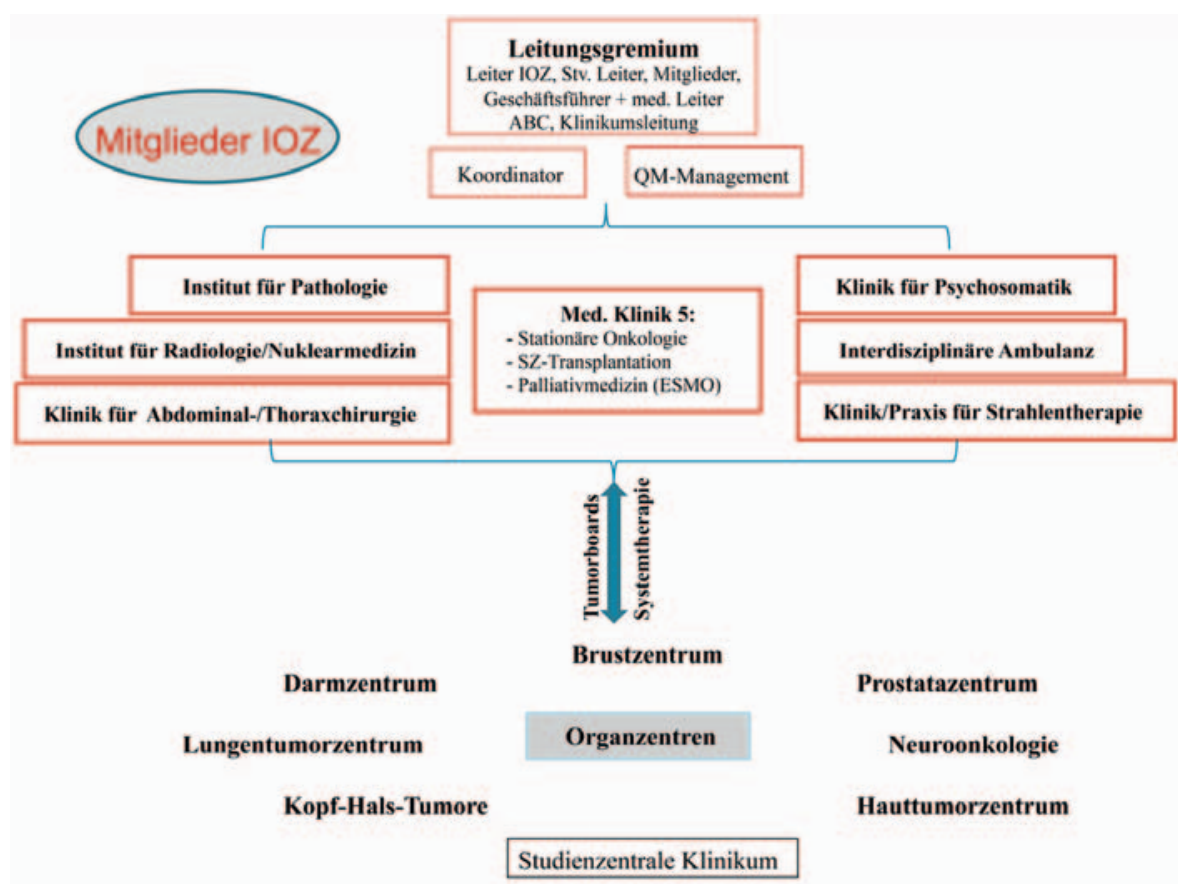


ser Kommission um ein unabhängiges «Kontrollgremium» aus Fachexperten handeln, das interdisziplinär zusammengesetzt ist. Die Tätigkeit der Kommission ist in einer Geschäftsordnung geregelt und ihre Entscheidungswege sind transparent darzustellen. Zweifellos sollte eine solche Kommission nur in begründeten Fällen Einfluss auf die Entscheidung des Zertifizierers nehmen, der ansonsten als «Herr des Verfahrens» anzusehen ist. Davon abzugrenzen ist eine Kommission, die die Qualitätsanforderungen als Voraussetzung für die Zertifizierung festlegt. Grundsätzlich können beide Kommissionen identisch sein oder sich aus unterschiedlichen Experten zusammensetzen.
Mit der Etablierung des Interdisziplinären Onkologischen Zentrums (IOZ) am Klinikum Nürnberg konnten wir die interne Struktur- und Prozessqualität weiter verbessern (Abb. 2). Mit der Vernetzung der onkologischen Kompetenz bieten wir den Patienten ein Behandlungskonzept auf hohem Niveau und mit kurzen Wegen.

\section{Disclosure Statement}

Es bestehen keine Interessenkonflikte. 